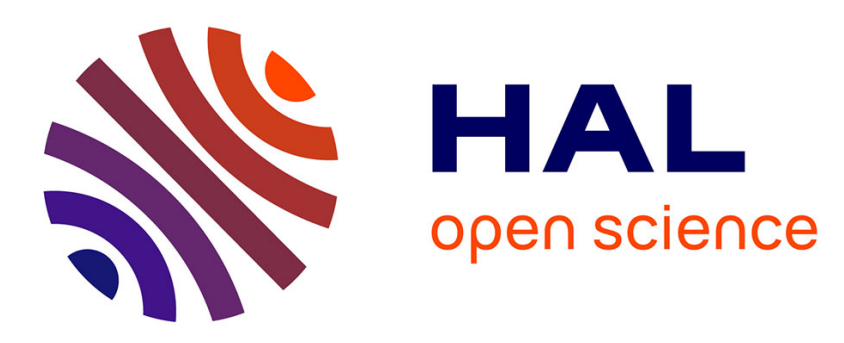

\title{
Multispectral device for help in diagnosis
}

Céline Delporte, Mohamed Ben Chouikha, Sylvie Sautrot, Françoise Viénot, Georges Alquié

\section{To cite this version:}

Céline Delporte, Mohamed Ben Chouikha, Sylvie Sautrot, Françoise Viénot, Georges Alquié. Multispectral device for help in diagnosis. Proceedings of SPIE, the International Society for Optical Engineering, 2012, Sensors, Cameras, and Systems for Industrial and Scientific Applications XIII, $8298,10.1117 / 12.909107$. hal-01565507

\section{HAL Id: hal-01565507 https://hal.science/hal-01565507}

Submitted on 19 Jul 2017

HAL is a multi-disciplinary open access archive for the deposit and dissemination of scientific research documents, whether they are published or not. The documents may come from teaching and research institutions in France or abroad, or from public or private research centers.
L'archive ouverte pluridisciplinaire HAL, est destinée au dépôt et à la diffusion de documents scientifiques de niveau recherche, publiés ou non, émanant des établissements d'enseignement et de recherche français ou étrangers, des laboratoires publics ou privés. 


\title{
Multispectral device for help in diagnosis
}

\author{
Céline Delporte*a , Mohamed Ben Chouikha*a, Sylvie Sautrot*b, \\ Françoise Viénot ${ }^{\mathrm{c}}$, Georges Alquié*a \\ ${ }^{\mathrm{a} U P M C}$ Univ Paris 06; \\ ${ }^{\mathrm{b}}$ Université Denis Diderot, Paris 07 \\ *UR 2, L2E, 4 place Jussieu, F-75005, Paris, France \\ ${ }^{c}$ Muséum National d'Histoire Naturelle - CRCC - CNRS (Paris, France)
}

\begin{abstract}
In order to build biological tissues spectral characteristics database to be used in a multispectral imaging system a tissues optical characterization bench is developed and validated. Several biological tissue types have been characterized in vitro and ex vivo with our device such as beef, turkey and pork muscle and beef liver. Multispectral images obtained have been analyzed in order to study the dispersion of biological tissues spectral luminance factor. Tissue internal structure inhomogeneity was identified as a phenomenon contributing to the dispersion of spectral luminance factor. This dispersion of spectral luminance factor could be a characteristic of the tissue. A method based on envelope technique has been developed to identify and differentiate biological tissues in the same scene. This method applied to pork tissues containing muscle and fat gives detection rates of $59 \%$ for pork muscle and $14 \%$ for pork fat.
\end{abstract}

Keywords: Multispectral imaging, biological tissues spectral characteristics database, help in diagnosis

\section{INTRODUCTION}

Biomedical optics has evolved in medical area as a new interdisciplinary field covering a wide range of therapeutic and diagnostic techniques [1]. One main advantage of optical techniques is the use of non-harmful radiation (excepted when UV radiation is used). Moreover optical techniques are often non-invasive, easier to use and cheaper to implement [2]. This field covers a wide range of therapeutic and diagnosis techniques like photodynamic therapy, laser surgery, thermotherapy, optical tomography, optical biopsy and in vitro diffuse spectroscopy. The two last methods use visible and near infrared spectroscopy technique. In particular optical biopsy is a non or minimally invasive spectroscopic analysis of biological tissue without cutting or removed tissues. In vitro diffuse spectroscopy requires relatively small samples and provides fast analysis [1]. Diffuse reflectance spectroscopy analyses and characterizes biological tissues in order to study and determine their optical properties [3]. Its biomedical applications include a wide variety of organs and tissue types like colon, esophagus, stomach, bladder, cervix, ovaries, breast, brain, liver, pancreas, heart, and oral tissues [4]. This technique can also diagnose various skin diseases, such as venous ulcers, skin necrosis and interstitial oedema for example [5]. However devices developed for these techniques allow a few measurement points number (typically 68) and optical properties may be biased by local tissue inhomogeneities [6].

Multispectral imaging is a powerful tool used in several application fields such as spatio-spectral diagnostics in agricultural [7], archaeology [8], art [9] and painting [10]. Multispectral imaging device determines the spectral characteristics of each pixel in the scene by providing images at several wavelengths. This method applied to the medical area may be a powerful tool for tissue analysis and may be an alternative solution to overcome optical spectroscopy limitations.

We are working on developing of a multispectral imaging system for intraoperative diagnosis aid purpose. The system should be able to identify and differentiate biological tissues in a surgical scene by measurement of their spectral reflectance in the visible and the near infrared wavelength range. In the other hand multispectral images in the near infrared may be used to identify buried tissues.

The architecture of our multispectral system is shown in Figure 1. The system is made up of two important parts, the acquisition system and the signal processing block. The acquisition system is constituted of a source that illuminates a scene. A CCD sensor records this scene at several wavelengths to give a multispectral image. The signal processing

Sensors, Cameras, and Systems for Industrial and Scientific Applications XIII, edited by Ralf Widenhorn, Valérie Nguyen, Antoine Dupret, Proc. of SPIE-IS\&T Electronic Imaging, SPIE Vol. 8298, 82980P - @ 2012 SPIE-IS\&T · CCC code: 0277-786X/12/\$18 · doi: 10.1117/12.909107 
block should be able to differentiate and identify biological tissues and diagnosis the state of the tissue. To fulfill this task this block has to use a biological tissues spectral characteristics database and an appropriate light propagation model.

A considerable literature exists on in vitro database with optical properties of a lot of biological tissues [11]. However these databases are developed with other techniques and the use of them is not adapted to multispectral imaging [12]. The aim of this paper is to develop a database including spectral responses of biological tissues characterized by multispectral imaging device.

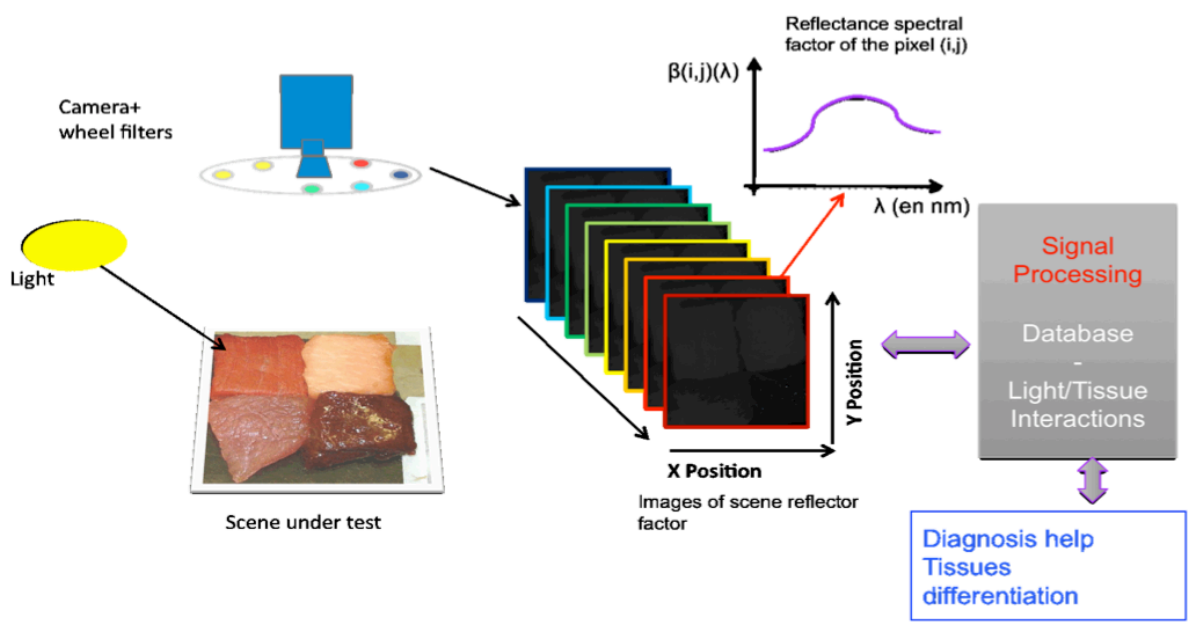

Figure 1. Architecture of multispectral imaging system for help in diagnosis. The system is made up of the acquisition system and the signal processing block.

A multispectral imaging device is developed to characterize biological tissues. In vitro and ex vivo measurements of several biological tissues have been carried out using this device. Spectral characteristics of these tissues will be analyzed and introduced.

\section{TISSUES OPTICAL CHARACTERIZATION BENCH BY MULTISPECTRAL IMAGING}

\subsection{Tests bench introduction}

The multispectral imaging test bench used for tissues characterization is shown in Figure 2. It is composed by one calibrated CCD camera, a monochromator with three holographic gratings and halogen light source.

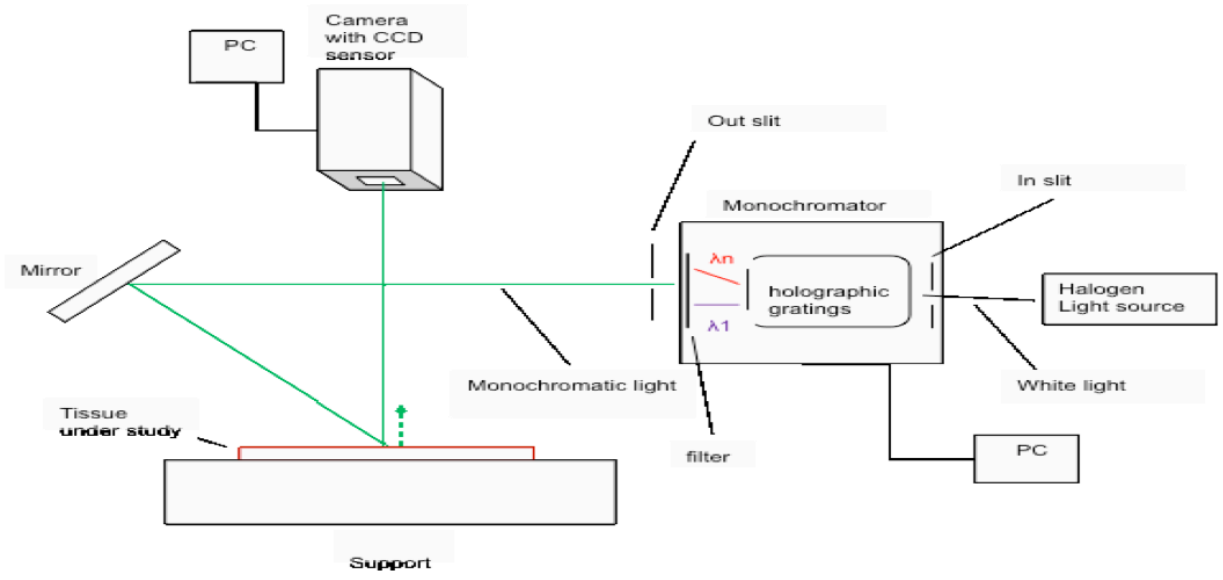

Figure 2. Architecture of optical characterization bench by multispectral imaging technique. 
The monochromator provides a narrow band radiation ray which illuminates the biological tissue. The tissue under study is fixed on a black box absorbing all wavelengths going through it. Multispectral images produced by this system are obtained according to the $0^{\circ} / 45^{\circ} \mathrm{CIE}$ standard geometry. In this case specular contribution is minimized. As the quality of multispectral images is linked to device characteristics the CCD sensor is calibrated to improve the accuracy of the measurements.

\subsection{CCD sensor calibration}

We set up a calibration bench to characterize CCD sensor using a monochromator and an integrated sphere. The CCD sensor under test is placed in front of the integrated sphere to be illuminated by uniform monochromatic light. In order to calibrate this sensor, the spectral response, the dynamic range and the noise were evaluated experimentally. After averaging images to correct the temporal noise, a difference from $10 \%$ to $20 \%$ between pixel responses is obtained due to the sensor Fixed Pattern Noise (FPN). We have developed an algorithm by establishing mathematical model of pixels response versus light intensity. In the case of this sensor a first order polynomial model is used to build a correction matrix in order to reduce FPN. Figure 3 (a.) shows an image of pixels response non-uniformity at $660 \mathrm{~nm}$. Figure 3 (b.) shows the image after correction at the same wavelength. FPN is reduced after the CCD sensor correction and the nonuniformity between pixels is decreased from $12 \%$ to $2 \%$.
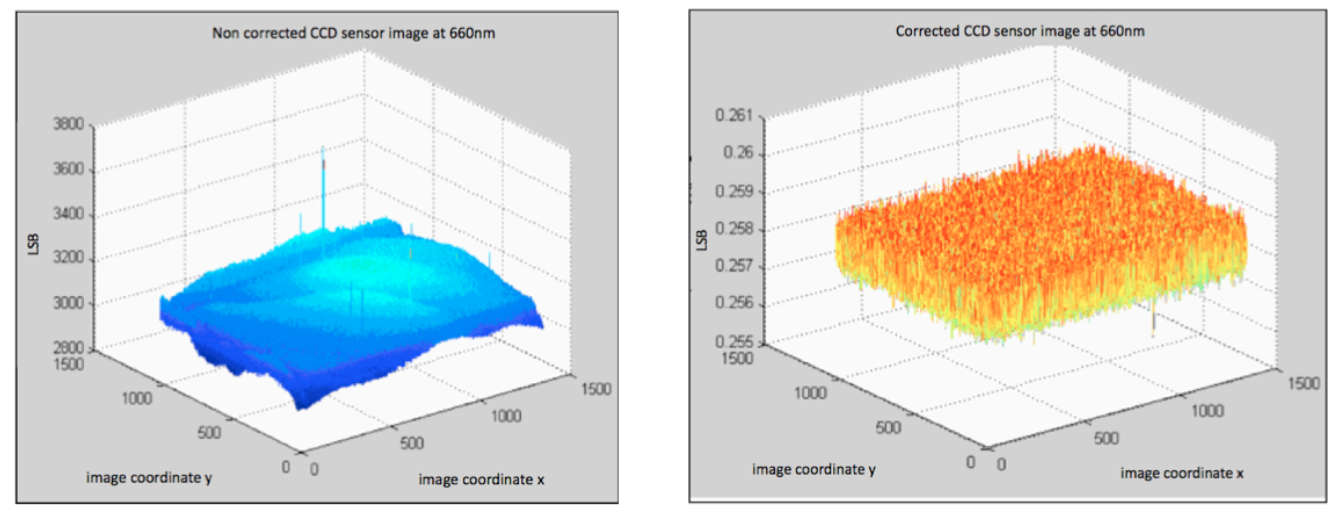

Figure 3. (a.) Image of pixels response non-uniformity at $660 \mathrm{~nm}$. FPN between pixels is about $12 \%$. (b.) Image after correction at $660 \mathrm{~nm}$. FPN between pixels is decreased to $2 \%$.

A calibrated CCD camera allows an accurate measurement of light distribution in the scene under study. We chose a 12 bits CCD camera in order to higher Signal to Noise Ratio (SNR).

An experimental protocol has been developed in order to characterize biological tissues in the same conditions. Tissues multispectral images are processed by a signal processing block to extract the spectral luminance factor $\beta(\lambda)$ of each image pixel.

\subsection{Image processing}

This image processing algorithm elaborated to extract the spectral luminance factor in a scene comprises two steps. The first step reduces the fixed pattern noise in the image with a correction matrix calculated during earlier calibration. The second calculates $\beta(\lambda)$ of each pixel for each corrected image using equation 1.

$$
\beta_{\text {Tissue }}(\lambda)=\frac{\rho(\lambda)_{\text {Tissue }}}{\rho(\lambda)_{\text {WhiteDiffuser }}}
$$

Where $\rho(\lambda)_{\text {Tissue }}$ is the object reflectance, $\rho(\lambda)_{\text {WhiteDiffiser }}$ the white diffuser reflectance.

The obtained multispectral image of the scene spectral luminance factor allows analysis of the spectral characteristics of each pixel. Figure 4 shows an example of spectral luminance factor cube of biological tissue and a plot of $\beta(\lambda)$ of one tissue area versus wavelength.

Once the CCD sensor is calibrated and the image processing is developed the biological tissue characterization bench needs to be validated. 


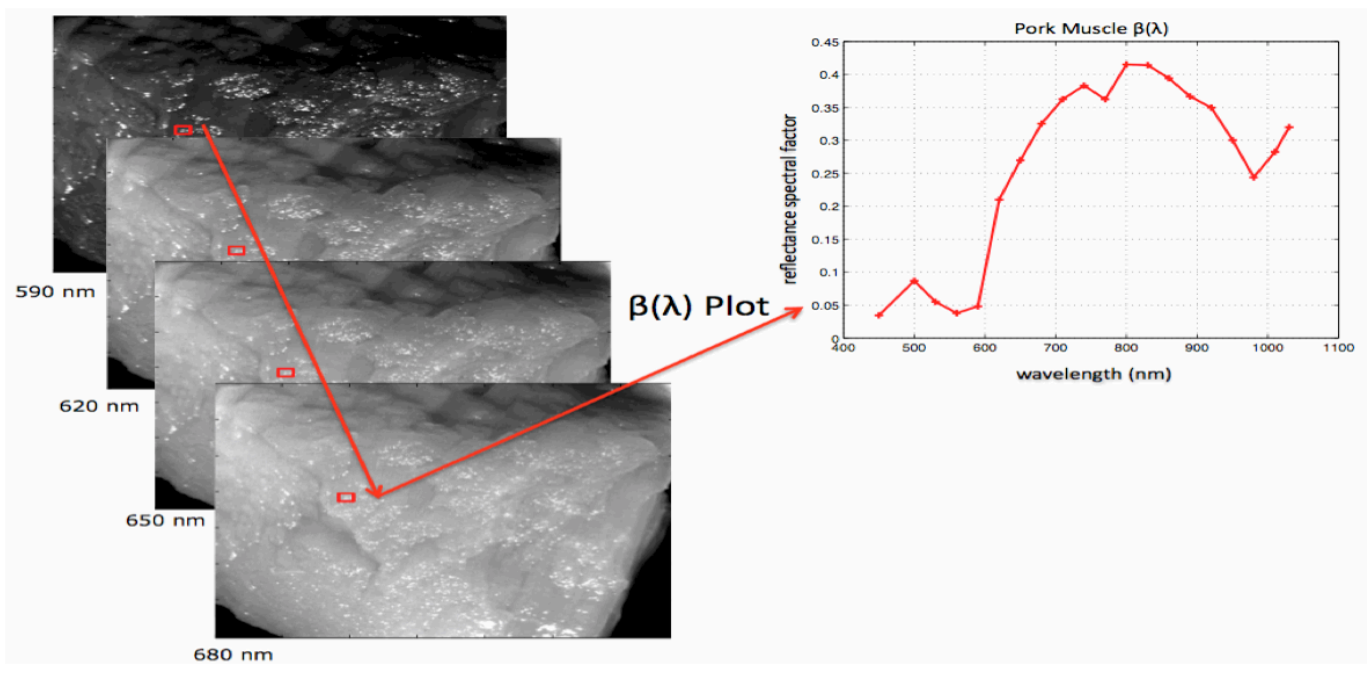

Figure 4. Spectral luminance factor example for a pork muscle area. Selected area is in red and its $\beta(\lambda)$ is plotted for every wavelength

\subsection{Device validation}

Measurements of spectral luminance factor of different gray and colored patches from the "CRISATEL" acrylic chart [13] have been carried out with our multispectral imaging characterization test bench and a spectrophotometer. The acrylic chart has been developed for multispectral imaging system characterization purpose.

Figure 5 (a.) shows the spectral luminance factor of a grey level patch obtained with the characterization test bench and with the spectrophotometer. A good agreement is obtained between characterization test bench measurements and those of the spectrophotometer. We defined a normalized dispersion error using equation 2 then calculated it on several uniform areas with different region sizes. Figure 5 (b.) shows dispersion error of spectral luminance factor as a function of the spectral luminance factor value at $570 \mathrm{~nm}$. As grey levels are homogeneous with uniform surface a relative dispersion error doesn't depend on size of window. However we observed that the higher the reflectance the lower the relative dispersion error. This shows that the relative dispersion error is closely related to Signal to Noise Ratio (SNR). At $570 \mathrm{~nm}$ relative dispersion error is lower than $4 \%$ for $\beta(\lambda)>0.3$.
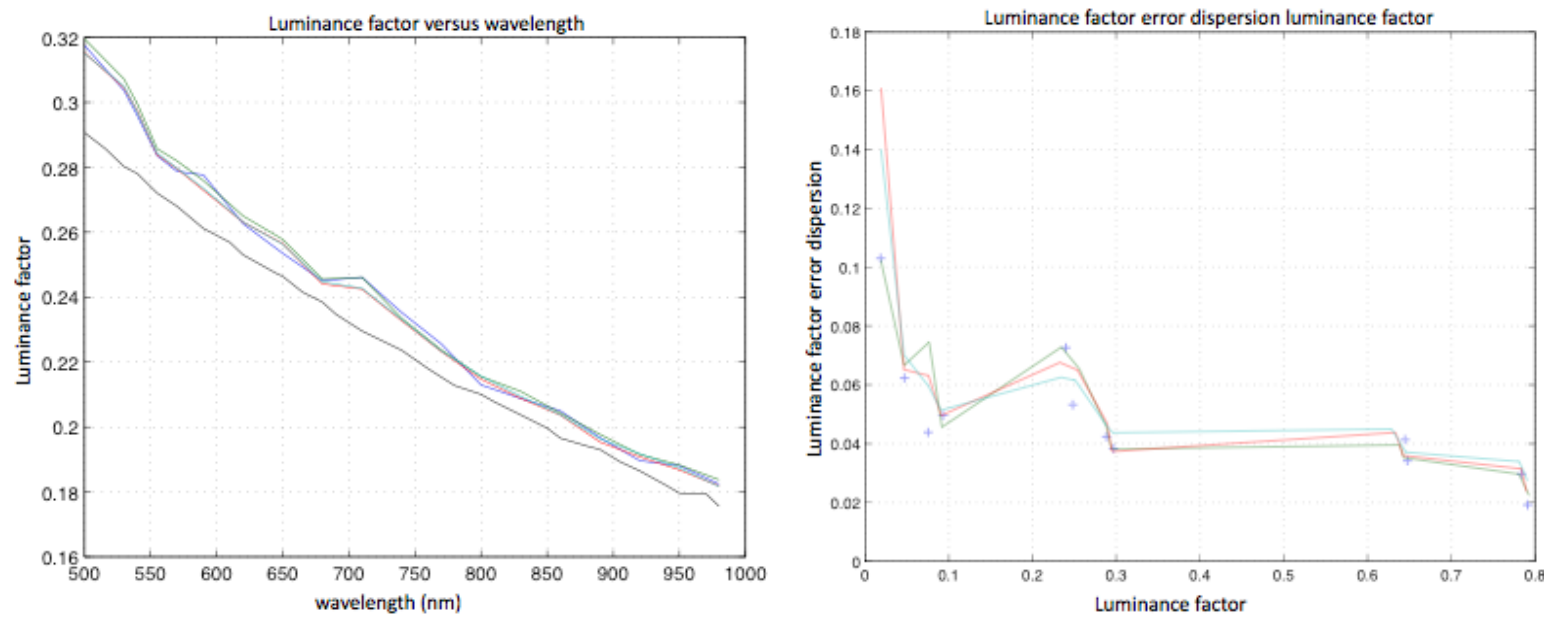

Figure 5. (a.) Spectral luminance factor versus wavelength for different size of window $(10 * 10$ pixels, $20 * 20$ pixels, $40 * 40$ pixels and $60 * 60$ pixels) with the characterization test bench and with the spectrophotometer. (b.) Dispersion error of spectral luminance factor versus spectral luminance factor value at $570 \mathrm{~nm}$ for different size of window ( $10^{*} 10$ pixels, $20 * 20$ pixels, $40 * 40$ pixels and $60 * 60$ pixels). 
These results validate the test bench measurements that can be used for biological tissues characterization.

\section{BIOLOGICAL TISSUES CHARACTERIZATION RESULTS}

Several biological tissues have been characterized with our multispectral imaging test bench. We made several tests with beef, turkey and pork muscle and beef liver in vitro and we characterized with our test bench 17 different tissues ex vivo on pork.

In order to study the spectral luminance factor $\beta(\lambda)$ of the tissue we analyzed biological tissues images. The next deals with the study of the spectral luminance factor of beef muscle, its variation from an area to another on the same tissue.

\subsection{Introduction of tissue optical characterization}

Beef muscle has been characterized with our multispectral imaging test bench. Luminance factor cube is obtained and Figure 6 (a.) shows the $\beta(\lambda)$ image at $660 \mathrm{~nm}$. We want to study the tissue spectral luminance factor $\beta(\lambda)$ and its variations on the tissue image. Twenty areas of 5 per 5 pixels are chosen on beef muscle image in order to calculate the spectral luminance factor median of each area. As we can see in Figure 6.a. the twenty areas are chosen in a homogeneous and similar texture regions. The plots of their luminance factor plots versus wavelengths in the $400 \mathrm{~nm}$ $1100 \mathrm{~nm}$ range are displayed in Figure 6 (b.).
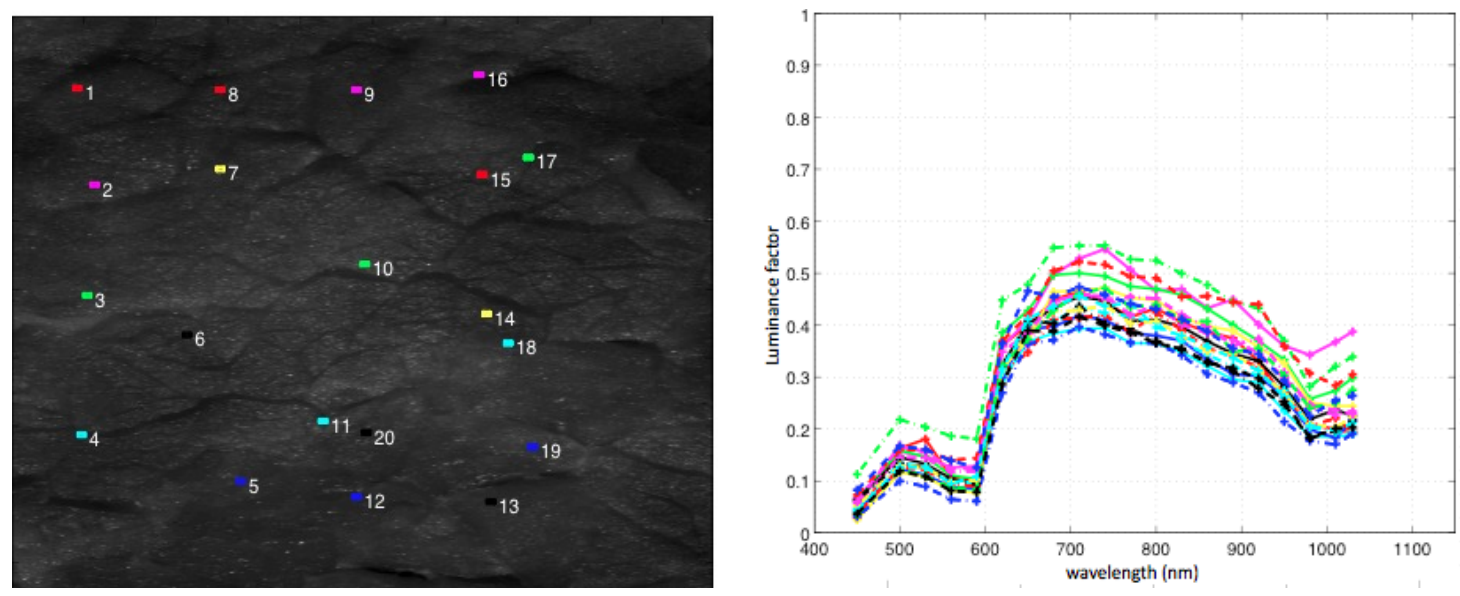

Figure 6. (a.) Beef muscle spectral luminance factor image at $660 \mathrm{~nm}$ with the $5 * 5$ pixels 20 areas studied on homogeneous region. (b.) $\beta(\lambda)$ of the 20 areas studied versus wavelength. $\beta(\lambda)$ of each area have same shape despite of their level difference

Spectral luminance factor medians for all of every area have same shape despite of their level difference.

We want to evaluate and quantify the variations of $\beta(\lambda)$ on the tissue. We define and calculate the normalized dispersion error $E_{D}$ and the normalized fidelity error $E_{F}$ for this purpose using equation 2 and equation 3 respectively.

$$
\begin{aligned}
& E_{D}(X(\lambda))=\frac{\sigma(X(\lambda))}{M(X(\lambda))} \\
& E_{F}(X(\lambda))=\frac{X \max (\lambda)-X \min (\lambda)}{M(X(\lambda))}
\end{aligned}
$$

Where $M(X(\lambda))$ is the median, $\sigma(X(\lambda))$ the standard deviation, $X \max (\lambda)$ is the maximal value and $X \min (\lambda)$ is the minimal value.

Figure 7 shows the $\beta(\lambda)$ dispersion error $E_{D}$ calculated on the twenty areas as a function of wavelength. Dispersion error $\mathrm{E}_{\mathrm{D}}$ is quite high. For wavelengths below $600 \mathrm{~nm}$ the dispersion error is higher than $20 \%$. $E_{D}$ decreases at $650 \mathrm{~nm}$ to reach $7 \%$ in order to increase from $680 \mathrm{~nm}$ to $1000 \mathrm{~nm}$ to reach an $E_{D}$ error of $20 \%$. 
Many factors may contribute to the high variation of $\beta(\lambda)$ on the tissue. Tissue surface roughness is probably an important factor. The luminance at the tissue surface may change from one area to another as shown in Figure 6.a. and this contributes to the dispersion $\beta(\lambda)$. We also suspect inhomogeneity of the tissue internal structure as a non-negligible factor.

Two analysis methods are developed in order to separate and identify error sources. These methods are introduced in the next section.

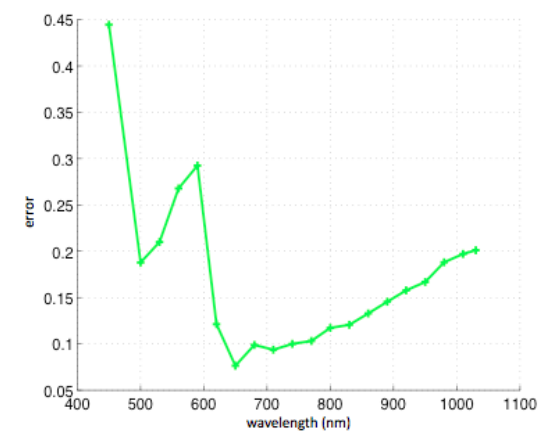

Figure 7. Dispersion error $E_{D}$ versus wavelength. $E_{D}$ is higher than $20 \%$ below $600 \mathrm{~nm}$ then decreases at $650 \mathrm{~nm}$ to reach $7 \%$ and increases from $680 \mathrm{~nm}$ to $1000 \mathrm{~nm}$ to reach $20 \%$.

\subsection{Incertitude sources evaluation methods}

In order to determine the influence of the tissue surface roughness we calculate $E_{D}$ on a tissue area and we study its variation as a function of the area. The influence of the tissue surface roughness has to be minimized with the increase of area. In order to determine the contribution of the non-homogeneity of the tissue surface luminance we developed a second method to calculate $E_{D}$. This method calculates the normalized dispersion error $E_{D}$ and the normalized fidelity error $E_{F}$ of one vector that comprises all of the twenty areas. We evaluated $E_{D}$ and $E_{F}$ variation as a function of area.

We applied the first method on twenty areas of the beef muscle of the Figure 6 (a.).
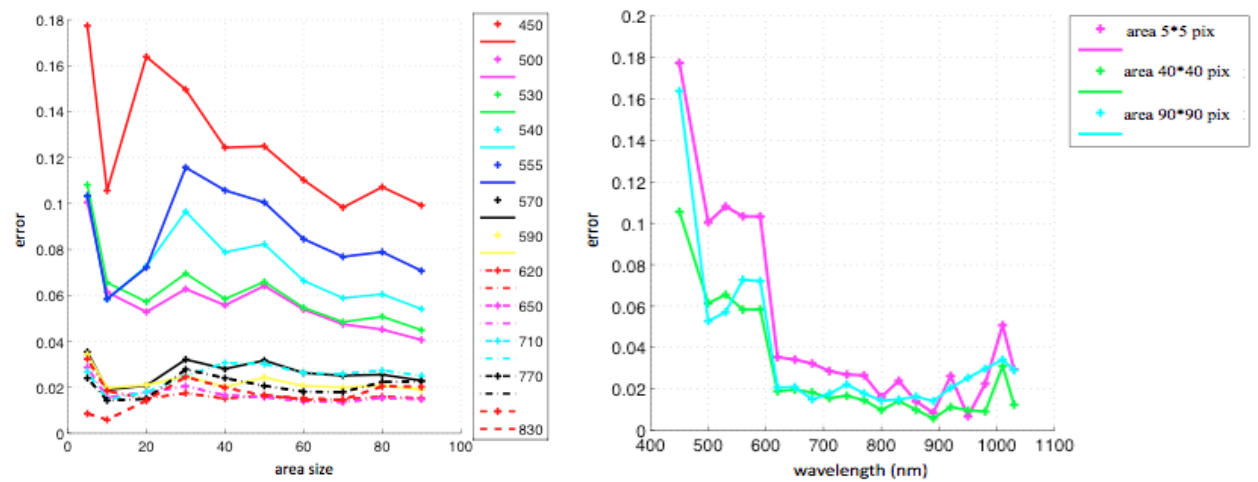

Figure 8. (a.) $E_{D}$ of an area versus area size for several wavelengths. $E_{D}$ decreases exponentially with area to reach a limit $E_{D r}$. (b.) $E_{D}$ of an area versus wavelength for several area sizes. $E_{D}$ decreases exponentially until $830 \mathrm{~nm}$ to reach $E_{D r}$.

We calculate $E_{D}$ versus area at each wavelength for each one of the twenty single areas. Figure 8 (a.) shows $E_{D}$ of an area as a function of area for several wavelengths. We note that $E_{D}$ decreases exponentially with the area so as to reach a limit $E_{D r}$. This confirms the idea of lowering the contribution of roughness to $E_{D}$ expanding the area. The final $E_{D r}$ limit may be caused by internal structure non-homogeneity of biological tissue.

Moreover Ed shows a non-negligible decrease with wavelength at each fixed area size as shown in Figure 8 (b.) This may be explained by the fact that small wavelengths radiation are absorbed at the tissue surface and can't go deeper in the tissue. This luminance factor $\beta(\lambda)$ at these short wavelengths is strongly affected by surface roughness, which increases $E_{D} . \beta(\lambda)$ is less affected by surface roughness because long wavelengths go deeper in the tissue. $E_{D}$ value at these wavelengths is essentially caused by the internal structure non-homogeneity of biological tissue. As a consequence 
$E_{D r}$ has different values in the red and near infrared range. We defined $E_{D r m a x}$ at $620 \mathrm{~nm}$ and $E_{D r m i n}$ at $830 \mathrm{~nm}$. We thought that the difference $\Delta E_{D r}=E_{D r \max }-E_{D r m i n}$ between these two limits is characteristic of tissue structure and nature.

In order to have a good insight of the contribution of $E_{D}$ of the tissue internal structure non-homogeneity we studied $E_{D r m i n}, E_{D r m a x}$ and $\Delta E_{D r}$ on the twenty areas of beef tissue.

Table 1 gives $E_{D r m i n}, E_{D r m a x}$ and $\Delta E_{D r}$ results calculated at each one of the twenty areas of Figure 6 (a.) These parameters show similar values from one area to another. A mean value of $1.05 \%$ is obtained for $E_{D r m i n}$ with a standard deviation of 0.4. $E_{D r m a x}$ mean value is of $2.65 \%$ with a standard deviation of $0.8 . \Delta E_{D r}$ mean value is $1.6 \%$ with standard deviation of 0.7 .

Table 1. $E_{D r m i n}, E_{D r m a x}$ and $\Delta E_{D r}$ results for the 20 areas.

\begin{tabular}{|c|c|c|c|c|c|c|c|c|c|c|}
\hline Area & $\mathbf{Z 1}$ & $\mathbf{Z 2}$ & $\mathbf{Z 3}$ & $\mathbf{Z 4}$ & $\mathbf{Z 5}$ & $\mathbf{Z 6}$ & $\mathbf{Z 7}$ & $\mathbf{Z 8}$ & $\mathbf{Z 9}$ & $\mathbf{Z 1 0}$ \\
\hline $\boldsymbol{E}_{\text {Drmin }}$ & $1 \%$ & $1 \%$ & $1 \%$ & $1 \%$ & $1 \%$ & $2 \%$ & $1 \%$ & $1 \%$ & $0 \%$ & $2 \%$ \\
\hline $\boldsymbol{E}_{\text {Drmax }}$ & $3 \%$ & $2 \%$ & $4 \%$ & $2 \%$ & $3 \%$ & $3 \%$ & $3 \%$ & $2 \%$ & $1 \%$ & $4 \%$ \\
\hline$\Delta \boldsymbol{E}_{\boldsymbol{D r}}$ & $2 \%$ & $1 \%$ & $3 \%$ & $1 \%$ & $2 \%$ & $1 \%$ & $2 \%$ & $1 \%$ & $1 \%$ & $2 \%$ \\
\hline
\end{tabular}

\begin{tabular}{|c|l|l|l|l|l|l|l|l|l|l|}
\hline Area & $\mathbf{Z 1 1}$ & $\mathbf{Z 1 2}$ & $\mathbf{Z 1 3}$ & $\mathbf{Z 1 4}$ & $\mathbf{Z 1 5}$ & $\mathbf{Z 1 6}$ & $\mathbf{Z 1 7}$ & $\mathbf{Z 1 8}$ & $\mathbf{Z 1 9}$ & $\mathbf{Z 2 0}$ \\
\hline $\boldsymbol{E}_{\text {Drmin }}$ & $1 \%$ & $1 \%$ & $1 \%$ & $1 \%$ & $1 \%$ & $1 \%$ & $1 \%$ & $1 \%$ & $1 \%$ & $1 \%$ \\
\hline $\boldsymbol{E}_{\text {Drmax }}$ & $3 \%$ & $3 \%$ & $2 \%$ & $3 \%$ & $2 \%$ & $3 \%$ & $2 \%$ & $2 \%$ & $4 \%$ & $2 \%$ \\
\hline$\Delta \boldsymbol{E}_{\boldsymbol{D r}}$ & $2 \%$ & $2 \%$ & $1 \%$ & $2 \%$ & $1 \%$ & $2 \%$ & $1 \%$ & $1 \%$ & $3 \%$ & $1 \%$ \\
\hline
\end{tabular}

Tissue internal structure inhomogeneity contributes to the normalized dispersion error. For this beef tissue we evaluated this contribution at $620 \mathrm{~nm}$ and its value is $E_{D r \max }=2.65 \%$. At $830 \mathrm{~nm}$ internal structure inhomogeneity contribution is $E_{\text {Drmin }}=1.05 \%$. The contribution of the tissue internal structure inhomogeneity is lower than the contribution of the surface roughness which can reach $20 \%$ at short wavelengths.

One disadvantage of this method to calculate $E_{D}$ arises from the fact that the enlargement of an area may increase $E_{D}$ instead of decreasing it. In fact by increasing the area we can overflow on tissue region with different brightness. This can't allow determining tissue internal contribution to dispersion error.

To overcome this limitation we used the second method to calculate $E_{D}$. In fact gathering the twenty areas in one vector could minimize the luminance fluctuations on the tissue surface. Thus we can lower the contribution of surface roughness by enlarging the area in order to reach the contribution of the tissue internal structure to $E_{D}$.

Figure 9 shows $E_{D}$ results calculated on a vector assembling all of the twenty areas of beef muscle. Figure 9 (a.) plots $E_{D}$ as a function of area at several wavelengths and Figure 9 (b.) plots $E_{D}$ as a function of wavelength for several areas. As observed earlier $E_{D}$ decreases exponentially when the area increases to reach a limit $E_{D r}$ that depends on wavelength. We observed that $E_{D}$ decreases between $620 \mathrm{~nm}$ and $700 \mathrm{~nm}$. We defined $E_{D r m a x}$ at $620 \mathrm{~nm}$ and $E_{D r m i n}$ at $700 \mathrm{~nm}$. Using this method $E_{D r m i n}$ equals $7 \%, E_{D r m a x} 11 \%$ and $\Delta E_{D r} 4 \%$. These Ed values are 4 to 7 times higher than those obtained with the first method.

In the case of this tissue the second method is not adapted to $E_{D}$ calculation. However the first method seems to be better adapted. In fact the tissue is flat allow sufficient widening of the studied area until it acceptably cancels the surface roughness effect. 

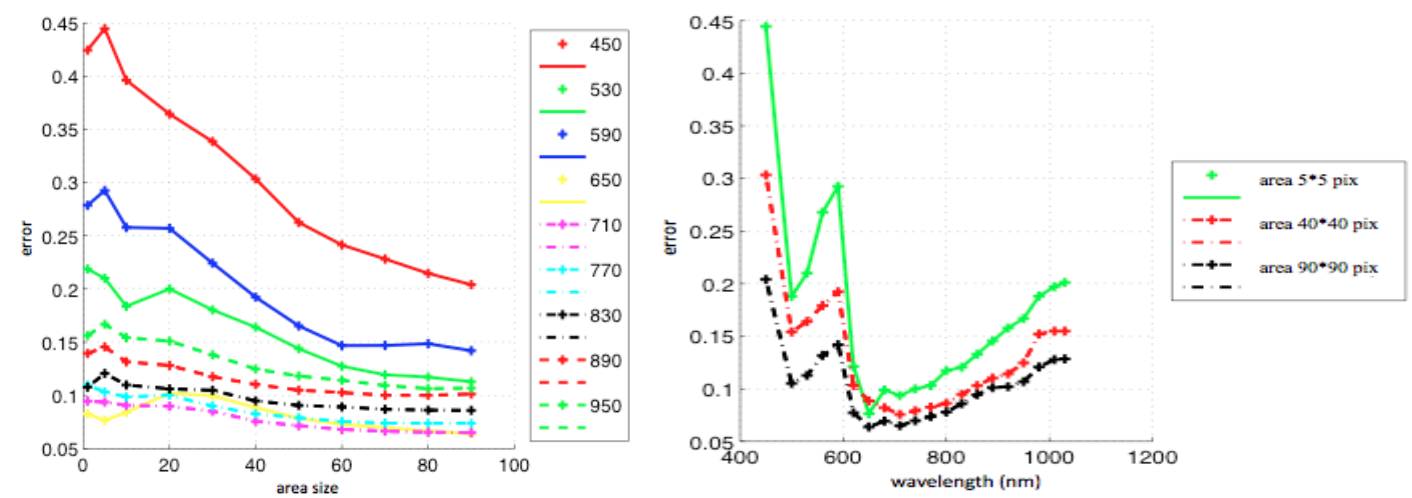

Figure 9. (a.) $E_{D}$ of the 20 areas versus area size for several wavelengths. $E_{D}$ decreases exponentially with area to reach a limit $E_{D r}$. (b.) $E_{D}$ of the 20 areas versus wavelength for several area sizes. $E_{D r}$ decreases exponentially until $700 \mathrm{~nm}$ to reach $E_{D r}$.

In order to quantify normalized fidelity error of $\beta(\lambda), E_{F}$ has been calculated with the second method.

Figure 10 (a.) shows $E_{F}$ of all of the twenty areas in function of area for several wavelengths and Figure 10 (b.) shows $E_{F}$ for all of the twenty areas in function of wavelength for several areas. Fidelity error shows the same trend as dispersion error. We defined $E_{F r m a x}$ at $620 \mathrm{~nm}$ and $E_{F r m i n}$ at $700 \mathrm{~nm}$. $E_{F r m i n}$ is of $20 \%, E_{F r m a x}$ of $40 \%$ and $\Delta E_{F r}$ of $20 \%$.
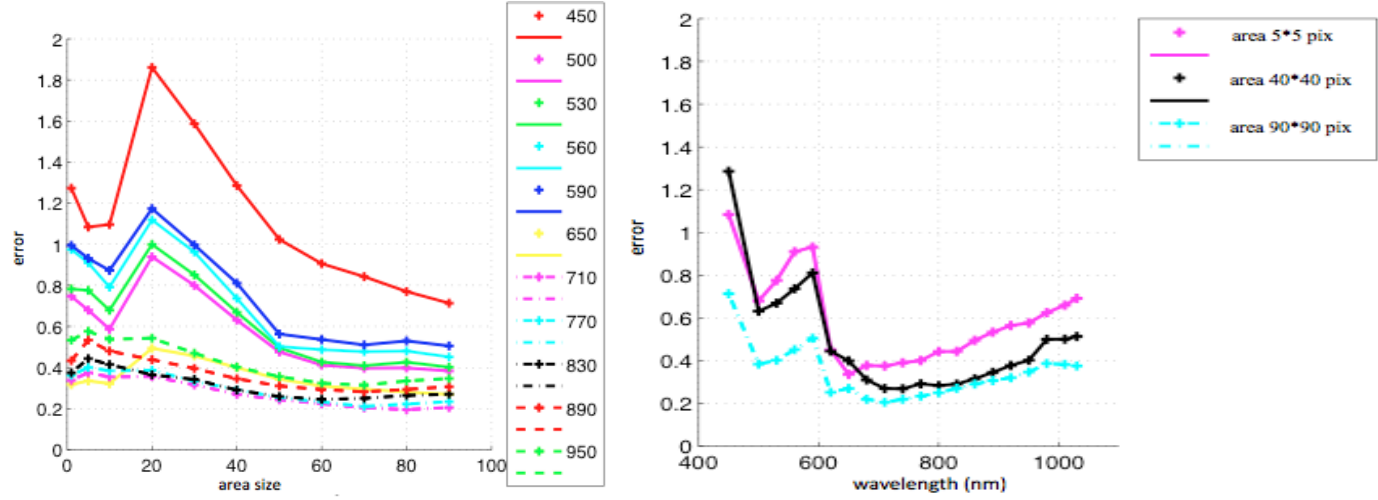

Figure 10. (a.) $E_{F}$ of an area versus area size for several wavelengths. $E_{F}$ decreases exponentially with area to reach a limit

$E_{F r}$. (b.) $E_{F}$ of an area versus wavelength for several area sizes. $E_{F}$ decreases exponentially until $700 \mathrm{~nm}$ to reach $E_{F r}$.

The dispersion error $E_{D}$ calculated with methods 1 and 2 and the fidelity error have enabled us to determine and quantify the phenomena contributing to $\beta(\lambda)$ variation on a tissue. One main phenomenon is the state of surface roughness inter alia. For short wavelengths, $\beta(\lambda)$ is strongly affected by surface roughness. In fact $E_{D}$ value can reach $20 \%$ with method 1 and $45 \%$ with method 2 . However when wavelengths go deeper in the tissue, $\beta(\lambda)$ is less affected by surface roughness.

The contribution of surface roughness can be cancelled with the expansion of the area. $\beta(\lambda)$ variation reaches a limit $E_{D r}$ defined by the non homogeneity of the tissue internal structure. We observed that $E_{D r}$ decreases with the wavelength and we defined $E_{D r m i n}$ and $E_{D r m a x}$ for each tissue. We think that these parameters and their difference $\Delta E_{D r}$ is a characteristic of the tissue. Beef muscle shows a $\Delta E_{D r}$ value of $1.6 \%$ with the first method and $4 \%$ with the second method. Internal structure error defines the limit of all measurements device as the imaging device that we want to develop. Moreover a reproducibility error could be added to these incertitude sources quantified by $E_{F}$ and its value can reach $20 \%$ for $\Delta E_{F r}$. An optimum size area for this tissue can be determined in order to minimize surface roughness contribution.

A statistical study was carried out on several tissue types such as beef, turkey and pork muscle and beef liver in order to evaluate $E_{D r m i n}, E_{D r m a x}$ and $\Delta E_{D r}$ variation from sample to sample and from one individual to another. 


\subsection{Results}

A statistical study has been made on several individuals for four types of tissue: beef muscle, turkey muscle, pork muscle and beef liver. Moreover this study will determine an optimum area in order to minimize the contribution of the surface condition to determine the spectral luminance factor for every types of tissue.

Tables 2, 3, 4 and 5 show $E_{D}$ calculated with the two methods and $E_{F}$ calculated with the second method respectively for beef muscle, turkey muscle, pork muscle and beef liver.

Table 2. $E_{D}$ and $E_{F}$ for beef muscle.

\begin{tabular}{|l|l|l|l|l|l|l|}
\hline & \multicolumn{2}{|c|}{$\begin{array}{c}E_{D} \\
\text { Method 1 }\end{array}$} & \multicolumn{2}{c|}{$\begin{array}{c}E_{D} \\
\text { Method 2 }\end{array}$} & \multicolumn{2}{c|}{$\begin{array}{c}E_{F} \\
\text { Method 2 }\end{array}$} \\
\hline & mean & std & mean & std & mean & std \\
\hline $\min$ & $3 \%$ & 2 & $5 \%$ & 1 & $21 \%$ & 4 \\
\hline $\max$ & $7 \%$ & 4 & $9 \%$ & 2 & $31 \%$ & 5 \\
\hline$\Delta$ & $5 \%$ & 2.5 & $3.5 \%$ & 2.5 & $11 \%$ & 4 \\
\hline
\end{tabular}

$\Delta E_{D r}$ determined with the second method is lower (3.5\%) than $\Delta E_{D r}$ calculated with the first method (5\%) and $E_{F}(11 \%)$.

Table 3. $E_{D}$ and $E_{F}$ for turkey muscle.

\begin{tabular}{|l|l|l|l|l|l|l|}
\hline & \multicolumn{2}{|c|}{$\begin{array}{c}E_{D} \\
\text { Method 1 }\end{array}$} & \multicolumn{2}{c|}{$\begin{array}{c}E_{D} \\
\text { Method 2 }\end{array}$} & \multicolumn{2}{c|}{$\begin{array}{c}E_{F} \\
\text { Method 2 }\end{array}$} \\
\hline & mean & std & mean & std & mean & std \\
\hline $\min$ & $4.5 \%$ & 4 & $5.75 \%$ & 3 & $21 \%$ & 9 \\
\hline $\max$ & $10 \%$ & 6.5 & $10 \%$ & 5 & $32 \%$ & 13 \\
\hline$\Delta$ & $5 \%$ & 3.5 & $5 \%$ & 3 & $11 \%$ & 7 \\
\hline
\end{tabular}

$\Delta E_{D r}$ determined with the second method is the same as $\Delta E_{D r}$ calculated with the first method (5\%) and both are lower than $E_{F}$ value (11\%).

Table 4. $E_{D}$ and $E_{F}$ for pork muscle

\begin{tabular}{|l|l|l|l|l|l|l|}
\hline & \multicolumn{2}{|c|}{$\begin{array}{c}E_{D} \\
\text { Method 1 }\end{array}$} & \multicolumn{2}{c|}{$\begin{array}{c}E_{D} \\
\text { Method 2 }\end{array}$} & \multicolumn{2}{c|}{$\begin{array}{c}E_{F} \\
\text { Method 2 }\end{array}$} \\
\hline & mean & std & mean & std & mean & std \\
\hline $\min$ & $2 \%$ & 1.5 & $5 \%$ & 2 & $17 \%$ & 6 \\
\hline $\max$ & $6 \%$ & 3.5 & $8.5 \%$ & 2.5 & $25 \%$ & 6 \\
\hline$\Delta$ & $4.5 \%$ & 7 & $3 \%$ & 1.5 & $9 \%$ & 3 \\
\hline
\end{tabular}

$\Delta E_{D r}$ determined with the second method is lower (3\%) than $\Delta E_{D r}$ calculated with the first method (4.5\%) and $E_{F}(9 \%)$. 
Table 5. $E_{D}$ and $E_{F}$ for beef liver

\begin{tabular}{|l|l|l|l|l|l|l|}
\hline & \multicolumn{2}{|c|}{$\begin{array}{c}E_{D} \\
\text { Method 1 }\end{array}$} & \multicolumn{2}{c|}{$\begin{array}{c}E_{D} \\
\text { Method 2 }\end{array}$} & \multicolumn{2}{c|}{$\begin{array}{c}E_{F} \\
\text { Method 2 }\end{array}$} \\
\hline & mean & std & mean & std & mean & std \\
\hline $\min$ & $0.7 \%$ & 0.7 & $10 \%$ & 6 & $20 \%$ & 8 \\
\hline $\max$ & $2 \%$ & 2.5 & $5.5 \%$ & 2.5 & $35 \%$ & 8 \\
\hline$\Delta$ & $2 \%$ & 2 & $4.5 \%$ & 4.5 & $13 \%$ & 5 \\
\hline
\end{tabular}

$\Delta E_{D r}$ determined with the first method is lower (2\%) than $\Delta E_{D r}$ calculated with the second method (4.5\%) and $E_{F}(13 \%)$.

$\Delta E_{D r}$ value determines the contribution of the internal structure for each tissue. It depends on the structure of the tissue. $\Delta E_{D r}$ is similar for beef $\left(\Delta E_{D r}\right.$ with method 1 equals $5 \%$ and $3.5 \%$ with second method), pork ( $\Delta E_{D r}$ with method 1 equals $5 \%$ and $5 \%$ with second method) and turkey ( $\Delta E_{D r}$ with method 1 equals $4.5 \%$ and $3 \%$ with second method) muscle. $\Delta E_{D r}$ value is the lowest (2\% with method 1 and $4.5 \%$ with method 2$)$ for beef liver. $\Delta E_{D r}$ could be characteristic for each tissue type in order to determine tissue nature.

This statistical study allows us to determine the contribution of internal structure error for each type of tissue. We will determine spectral luminance factor for each type of these tissues under study minimizing contribution of surface condition.

\subsection{Spectral characteristics of biological tissues}

In order to identify biological tissues, a database has to be developed with all the biological tissues spectral characteristics. These spectral characteristics take into account different incertitude sources developed earlier. A method was developed in order to define luminance factor in minimizing the contribution of surface roughness. This method is based on the calculation of the tissue envelope.

This method consists in determining the histogram of each area under study for every wavelength. Gaussian normal law expectation and standard deviation are then determined from the histogram for each wavelength. Standard deviations composed the envelope. Envelope mean is given by Gaussian expectation of $\beta(\lambda)$. The Figure 11 gives an example of beef muscle envelope for one area size (Figure 6. (a.)).

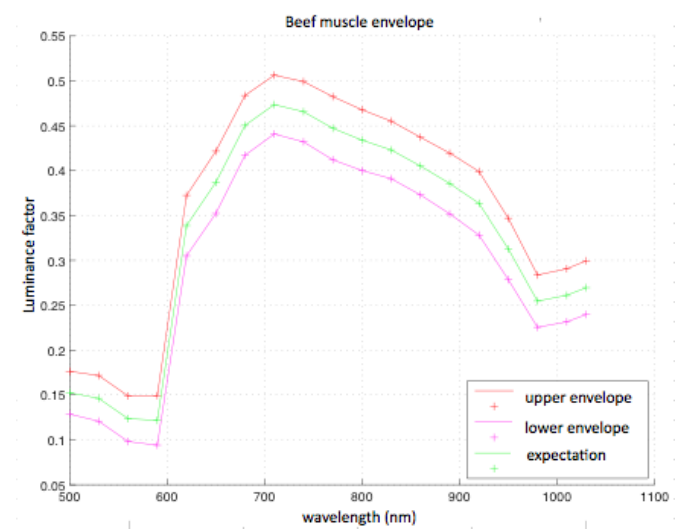

Figure 11. Beef muscle envelope for 50*50 pixels area. This envelope is the spectral characteristic for this tissue. Area size is chosen to minimize roughness surface state of tissue

As we have selected the optimum area, the so determined envelope minimizes the error due to surface condition. Envelopes for every type of biological tissue can be determined for each individual. Figure 12 shows $\beta(\lambda)$ expectation for beef, turkey and pork muscle and beef liver of one characterized scene. 


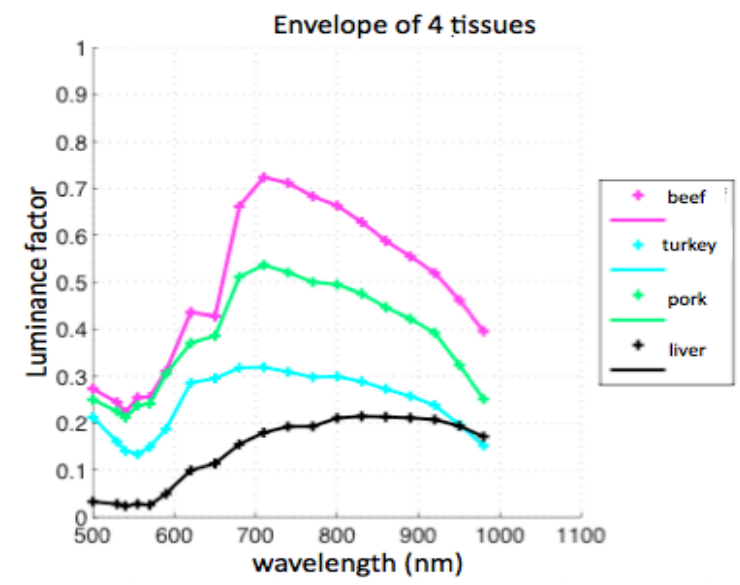

Figure 12. Beef, turkey and pork muscle and beef liver envelopes of one characterized scene. Beef liver envelope is clearly different of the others. The three other tissue envelopes are similar despite small differences between them.

Beef liver $\beta(\lambda)$ shape shows a quite difference from three others biological tissues. Even if their shapes are different the three others shapes are similar to each other. We have to identify tissues even if they are similar. Moreover errors determined are high and we have to consider these errors to identify and differentiate biological tissues.

\section{BIOLOGICAL TISSUES DIFFERENTIATION}

The aim of database elaboration is to identify and differentiate biological tissues that are presents on the same scene. For every biological tissue characterized by our device its enveloped has been determined. In order to differentiate tissues for a first time a first solution is to determine the spectral luminance factor for one area and to compare it to a tissue envelope in order to know whether this area belongs to the tissue or not. One example is shown in Figure 13 with pork muscle.
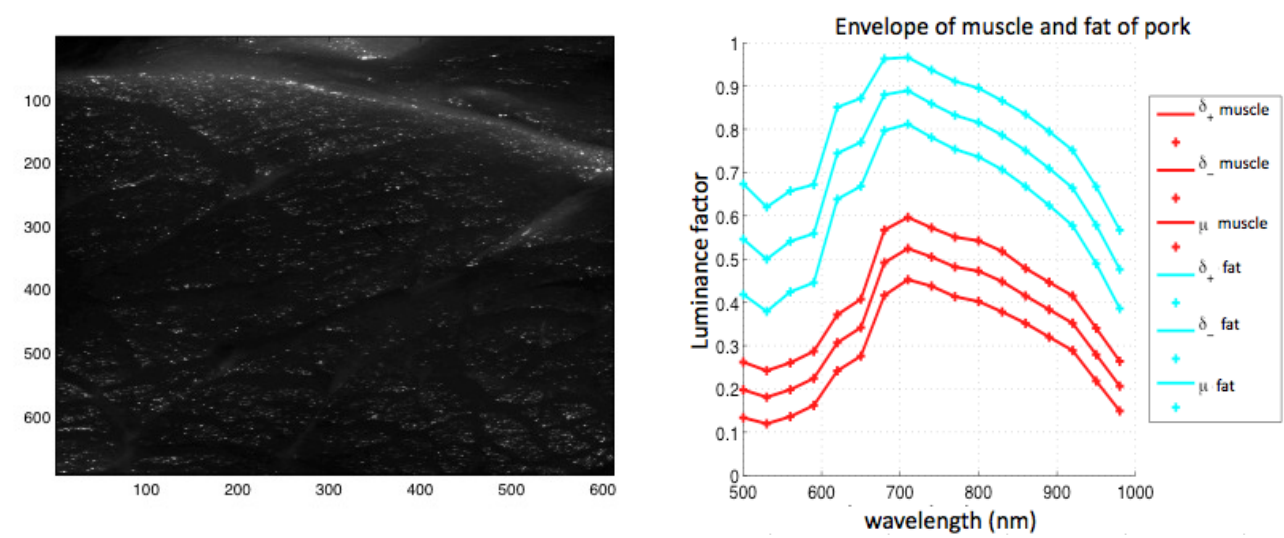

Figure 13. (a.) Pork muscle $\beta(\lambda)$ at $650 \mathrm{~nm}$. Flat pork is on the image top in white and muscle is gray. (b.) Pork muscle envelope and pork fat envelope. Envelops exhibit the same shape despite their vertical displacement

The image covering a 5 per 5 pixels window. $\beta(\lambda)$ median of this window is compared with muscle and fat envelopes. The more frequently $\beta(\lambda)$ median belongs to the envelope the more likely the pixel belongs to this tissue.

Figure 14 shows probability for each pixel to belong to pork muscle (a.) and pork fat (b.). 

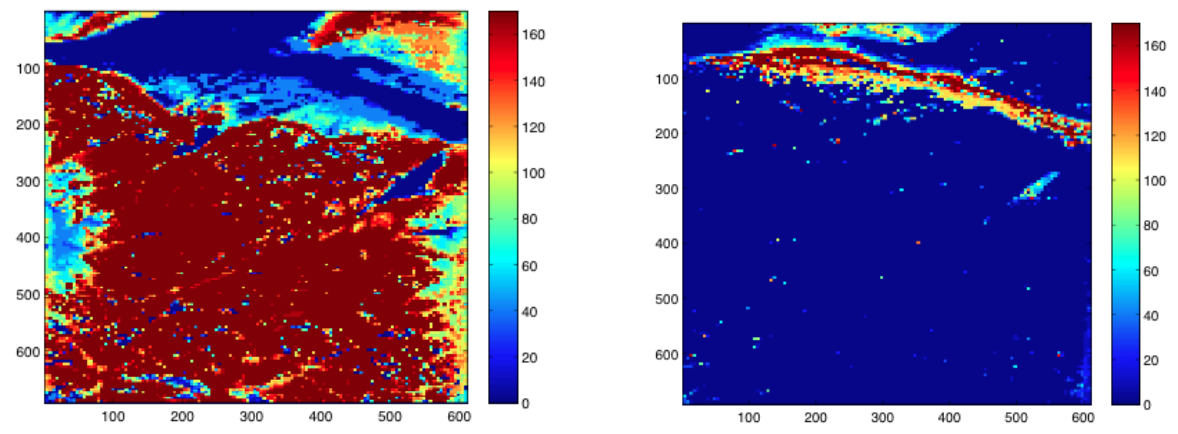

Figure 14. (a.) Probability for each pixel to belong to pork muscle. The higher the pixel is red, the more it belongs to pork muscle. (b.) Probability for each pixel to belong to pork fat. The higher the pixel is red, the more it belongs to pork fat.

If $\beta(\lambda)$ median is entirely inside the envelope, the detection rate is $59 \%$ for pork muscle and $14 \%$ for the pork fat. The detection rate is low for pork fat and not enough high for pork muscle. This technique for differentiating the tissues has to be improved in order to increase the detection rate for each tissue. Moreover if the envelope level changes between one individual to another, then the detection rate will be low because the database will not be the same for several samples of the tissue type.

\section{CONCLUSION AND PERSPECTIVES}

The aim of this work is to develop a biological tissues spectral characteristics database to be used in a multispectral imaging system. We set up a test bench using a calibrated CCD sensor and a monochromator for tissue characterization by multispectral imaging technique. The test bench has been validated by carrying measurements of the CRISATEL acrylic chart samples and by comparing the results with those of a spectrophotometer. Once test bench had been calibrated, several biological tissues were characterized in vitro such as beef, turkey and pork muscle and beef liver. Ex vivo characterization was also conducted in removing seventeen tissues on a living pork in animal laboratory.

Multispectral images were analyzed with an image processing tool to study the variation of the spectral luminance factor $\beta(\lambda)$ on the same tissue. We observed $20 \%$ to $30 \% \beta(\lambda)$ variation on a beef muscle. This high $\beta(\lambda)$ variation on a given tissue may be an obstacle to identify and differentiate biological tissues.

We developed an analysis technique to determine and quantify the contribution of different phenomena involved in this variation. One main factor is the tissue surface roughness and its contribution to beef muscle $\beta(\lambda)$ variation was evaluated with our analysis technique. We found that the contribution of the surface roughness is depending upon the wavelength and can reach $20 \%$ at short wavelengths. We identified the inhomogeneity of the tissue internal structure as a factor contributing to $\beta(\lambda)$ variation. Its contribution is wavelength dependent and decreases with increasing wavelength. This factor contribution has been quantified for beef muscle and its value is $1.6 \%$. The non-uniformity of the tissue surface luminance is also a factor that contributes to $\beta(\lambda)$ variation.

Image processing can be used to minimize the contribution of the non-uniformity of the tissue surface luminance and of the surface roughness to $\beta(\lambda)$. However the non-homogeneity of the tissue internal structure defines the limit of all measuring system as the imaging device that we want to develop.

We think that the non-homogeneity of the tissue internal structure can be considered as a tissue characteristic. As the dispersion of the normalized $\beta(\lambda)$ decreases with increasing wavelength, we defined $E_{D r m i n}$ as the normalized $\beta(\lambda)$ dispersion at $830 \mathrm{~nm}$ and $E_{D r \max }$ as the normalized $\beta(\lambda)$ dispersion at $620 \mathrm{~nm}$. The difference $\Delta E_{D r}=E_{D r \max }-E_{D r \min }$ could be an additional parameter characteristic of the biological tissue. In order to verify these assumptions a statistical study was carried out on several tissue types such as beef, turkey and pork muscle and beef liver in order to study $\Delta E_{D r}, E_{D r m i n}$ and $E_{D r m a x}$ variation from sample to sample and from one individual to another. We observed that the three parameters have shown a variation from sample to sample on the same individual and from one individual to another. We calculated the mean values and we found $E_{D r m i n}=3 \%, E_{D r \max }=7 \%, \Delta E_{D r}=5 \%$ for beef muscle, $E_{D r m i n}=4.5 \%, E_{D r m a x}=10 \%, \Delta E_{D r}=5 \%$ for turkey muscle, $E_{D r \min }=2 \%, E_{D r \max }=6 \%, \Delta E_{D r}=4.5 \%$ for pork muscle and $E_{D r \min }=0.7 \%, E_{D r \max }=2 \%, \Delta E_{D r}=2 \%$ for beef liver. $E_{D r m i n}, E_{D r m a x}$ and $\Delta E_{D r}$ of beef and pork muscle have closely related values due to the fact that their structures are similar. Even if $E_{\text {Drmin }}, E_{\text {Drmax }}$ of turkey muscle are close to those of the two other types of muscle we observed a 
difference with this tissue and $\Delta E_{D r}$ is the same. This may be explaining by the fact that the three tissues belong to the same class i.e. muscle. In other hand $E_{D r m i n}, E_{D r \max }$ and $\Delta E_{D r}$ of beef liver are different and lower values than those of muscle.

In order to use results of biological tissues characterization to build a biological tissues spectral characteristics database, we proposed a method to identify and differentiate biological tissues. This method consists in determining the $\beta(\lambda)$ envelope of a tissue minimizing the contribution of surface roughness. One study on pork muscle and fat was made. We obtained a moderate detection rate for muscle with $59 \%$ and a low rate for fat with $14 \%$. This method could be improved in order to have a better detection of each tissue. In fact we could use additional parameters for detecting tissue as the tissue internal structure and others parameters which are under study.

Compared to single point spectral characterization of tissue, spectral imaging is a promising technique.

\section{REFERENCES}

[1] Jan Sorensen Dam, [Optical analysis of biological media - continuous wave diffuse spectroscopy], Doctoral thesis, Lund Institute of Technology, 15-16 (November 2000).

[2] Pieter van der Zee, [Measurement and modeling of the optical properties of human tissue in the near infrared], Doctoral thesis, University College London, 22 (December 1992).

[3] Erick Häggblad, [In vivo diffuse reflectance spectroscopy of Human tissue: From point measurements to imaging], Doctoral thesis, Liköping University, 11-12 (2008).

[4] G. Zonios and A. Dimou, "Modelling diffuse reflectance from semi-infinite turbid media: application to the study of skin optical properties", Optics Express, Vol. 14, Issue 19, 8661-8674 (2006).

[5] I.V. Meglinski, S.J. Matcher, "Computer simulation of the skin reflectance spectra", Computer Methods and Programs in Biomedicine, Vol. 70, Issue 2, 179-186 (2003).

[6] A. Kienle, L. Lilge, M. S. Patterson, R. Hibst, R. Steiner, and B. C. Wilson, "Spatially resolved absolute diffuse reflectance measurements for noninvasive determination of the optical scattering and absorption coefficients of biological tissue", Applied Optics, Vol. 35, Issue 13, 2304-2314 (1996)

[7] L. C. Lobo, O. K. Ersoy, G. E. Miles, [Multispectral imaging, image processing and classification for agriculture], ECE Technical Reports. Paper 20 (2000).

[8] Haida Liang, "Advances in multispectral and hyperspectral imaging for archaeology and art conservation", Applied Physics A: Materials Science \& Processing, 1-15 (2011).

[9] G. A. Ware, D. M. Chabries, and R. W. Christiansen, "Multispectral Document Enhancement: Ancient Carbonized Scrolls", Geoscience and Remote Sensing Symposium 2000 Proceedings IGARSS 2000 IEEE 2000 International, Vol. 6, 2486-2488 (2000).

[10] A. Ribés Cortés, [Multispectral Analysis and Spectral Reflectance Reconstruction of Art Paintings], Doctoral thesis, Ecole Nationale Supérieure des Télécommunications (2003).

[11] S. J. Matcher, M. Cope, and D. T. Delpy, "In vivo measurements of the wavelength dependence of tissuescattering coefficients between 760 and $900 \mathrm{~nm}$ measured with time-resolved spectroscopy", Applied Optics, Vol. 36, Issue 1, 386-396 (1997)

[12] W.F. Cheong, S.A. Prahl, A.J. Welch, "A review of the optical properties of biological tissues", IEEE Journal of Quantum Electronics, Vol. 26, Issue 12, 2166 - 2185 (1990).

[13] http://www.c2rmf.fr/pages/page id18484_u112.htm 